\title{
Diagnostic Support System for the Detection of Attention Deficit Hyperactivity Disorder Using EOG Signals
}

\section{Esra Demirci}

Erciyes University: Erciyes Universitesi

\section{Mustafa Yasin Esas}

Erciyes University: Erciyes Universitesi

Çiğdem Gülüzar Altıntop

Erciyes University: Erciyes Universitesi

\section{Neslihan Taştepe}

Erciyes University: Erciyes Universitesi

Fatma Latifoğlu ( $\nabla$ flatifoglu@erciyes.edu.tr)

Erciyes University https://orcid.org/0000-0003-2018-9616

\section{Research Article}

Keywords: Electrooculogram EOG, Visual stimulus test, Attention Deficit Hyperactivity Disorder, ADHD, Artificial Neural Networks, ADHD Diagnostic Support System

Posted Date: May 24th, 2021

DOl: https://doi.org/10.21203/rs.3.rs-462902/v1

License: (c) (1) This work is licensed under a Creative Commons Attribution 4.0 International License. Read Full License 


\title{
DIAGNOSTIC SUPPORT SYSTEM FOR THE DETECTION OF ATTENTION DEFICIT HYPERACTIVITY DISORDER USING EOG SIGNALS
}

\author{
${ }^{1}$ Esra Demirci \\ esrademirci@erciyes.edu.tr \\ ${ }^{2}$ Mustafa Yasin Esas \\ mustafaesas@gmail.com \\ ${ }^{2}$ Çiğdem Altıntop \\ cigdemacer@erciyes.edu.tr \\ ${ }^{1}$ Neslihan Taştepe \\ ntastepe1@ hotmail.com \\ ${ }^{2}$ Fatma Latifoğlu \\ flatifoglu@erciyes.edu.tr
}

${ }^{1}$ Department of Child and Adolescent Psychiatry, Erciyes University School of Medicine, Turkey

${ }^{2}$ Department of Biomedical Engineering, Engineering Faculty, Erciyes University, Turkey

\section{Correspondence Address}

Assoc. Prof. Dr. Fatma LATİFOĞLU

Erciyes University, Engineering Faculty

Biomedical Engineering Dept.

39039, Kayseri/TURKEY

E-mail :flatifoglu@erciyes.edu.tr

Phone : + 90 (352) 2076666 extension: 32377 


\begin{abstract}
-
Although Attention Deficit Hyperactivity Disorder (ADHD) is a common childhood disease, objective diagnostic methods are insufficient still. Current diagnostic methods include the subjective influence of the evaluator. In this context, in our study, we aimed to minimize the subjective effect of the evaluator with the objective diagnosis support system for ADHD.

In our study, a visual stimulus follow-up test developed by us was applied to the patient with ADHD and healthy individuals, and electrooculogram (EOG) signals were recorded simultaneously. With the features extracted from EOG signals, Artificial Neural Networks (ANN) were used for the classification study of patients and healthy individuals, and it was determined that the classification of ADHD and healthy group could be distinguished by $81.76 \%$ performance. Thus, the outcomes that will contribute to the objective diagnosis of ADHD have been presented. The results are remarkable and important findings have been obtained that will contribute to the literature.
\end{abstract}

Keywords: Electrooculogram EOG, Visual stimulus test, Attention Deficit Hyperactivity Disorder, ADHD, Artificial Neural Networks, ADHD Diagnostic Support System 


\section{INTRODUCTION}

Attention deficit hyperactivity disorder (ADHD) is one of the neuropsychiatric diseases that are common especially in childhood [1,2]. According to the DSM-IV, DSM-V (Diagnostic and Statistical Manual of Mental Disorders) criteria, which is the international classification system, this disease; There are three basic types: attention deficit, hyperactivity and impulse control, and the combined type [3]. It is considered that the main problem in ADHD is caused by the inadequacy of mental functions as well as the state of hyperactive [4]. Since ADHD mostly manifests itself in pre-school or early years of school, it causes deterioration and lack of development in academic, social and professional areas from early ages, and accompanying psychiatric problems [5]. ADHD is mostly seen and detected in the 8-13 age group, and literature studies show that the prevalence of ADHD in the population is close to 20\% [6-9]. Psychometric tests are generally used in ADHD diagnosis and treatment process. However, there is no objective assessment tool and diagnostic support system that would eliminate the subjectivity of the person applying the tests for ADHD or minimize its effects. The following procedures is performed by psychiatrists and psychologists during the diagnosis and treatment of ADHD. WCST [10], Stroop Test [11] and Line Orientation Test [13] is applied to the patient and control groups. IQ measurements is made with the WISC-R [12] test. The Conners Rating Scale-Parent [14] and Teacher Form [15] and the Clinical Global Follow-up Rating Scale is used to follow-up the response to treatment. (The content and details of these tests can be found in reference sources.

In our study, it is aimed to determine ADHD with objective approach using electrooculogram (EOG) signals to determine the ADHD and healthy subject.

EOG is an electrical magnitude resulting from obtaining the electrical potential change of the eyeball by using electrodes, depending on the movements of the eyeball. EOG can provide information about eye movements, electrical activity in the eye, and visual pathway diseases [16]. When literature studies on ADHD and EOG were reviewed, no study based on EOG signals for ADHD diagnosis was found. However, studies using EOG signals and some recent studies on ADHD are presented below.

A study on ADHD symptoms included 170 adolescent individuals with a mean age of 16.9. EEG signals during and after sleep were studied and the effects of ADHD symptoms on learning were examined by applying a memory test. It has been found that individuals with ADHD symptoms show low learning. [17]. 
In a study, event-related potential (ERP) signals from ADHD and schizophrenia patients were analyzed. Behaviors of subjects and EEG data were recorded from 132 healthy controls and 63 ADHD and 68 schizophrenia patients with similar age, gender, and educational status. With this study, it was determined that the latent ERP component approach is suitable for revealing functional impairment in ADHD and schizophrenia and that there are qualitatively different cognitive deficit patterns in ADHD and schizophrenia [18].

In a study on motor skills using EEG signals in children with ADHD, it was evaluated that motor skills control the relationship between vigorous physical activity and resting-state [19].

A study was carried on the classification of ADHD and non-ADHD individuals using the Universal Background model. It was stated that the likelihood ratio detector could be designed to define the ADHD and Universal Background model using the Gaussian mixture model. As a result of that study, based on a limited database, it has been determined that when using EEG signals recorded during an attention network task, high detection, and the low error rate can be obtained simultaneously with the proposed approach, this classification can be made with this method [20].

Machine learning-based classification of individuals with ADHD and non-ADHD using the time/frequency characteristics of event-related neuroelectric activity was peirformed in a literatüre study. Time-frequency domain characteristics of EEG event-related potential (ERP) signals recorded during the Stroop task were analysed. Support Vector Machine is used to obtain the best distinguishing features. When the best three features and five features were used, it was found that the classification accuracy for the training dataset increased to $98 \%$ and $99.5 \%$ respectively. Accuracy for the test dataset was 100\%. Based on extensive experiments, the delta band of the EEG signals showed the most distinctive frequency band with statistical parameters emerging as the most contributing feature set [21].

In a literature study, on performance analysis of univariate and multivariate EEG signals in the diagnosis of ADHD, EEG from 8 cortical regions was recorded from 22 combined type ADHD patient and 21 healthy male controls (age range 4-15 years) with eyes open and closed. Univariate and interdependence measurements calculated from the frequency domain and the reconstructed state domains of the EEG signals were calculated. The performance in distinguishing those with ADHD from healthy individuals was analyzed. It has been evaluated that ADHD individuals can be classified with high accuracy [22]. 
In a study on determination of gender in adolescent ADHD, EEG and electrodermal activity (EDA) signals were recorded simultaneously. It is seen that, theta activity of the EEG signals in men with ADHD is increased and EDA signals in women with ADHD are decreased [23].

Ability to concentrate and problem solving factors were evaluated in a study. Blink speed (for focus), blink strength, and head posture were calculated. In this study, the relationship between two psychological responses and the EOG parameter was investigated. The qualitative correlation between the EOG parameter, which corresponds to the concentration ability, and the psychological response can be verified, and it is predicted that it can be applied to elearning in virtual classrooms in the near future [24].

Automatic scoring of sleep stages was performed in a study on sleep stage classification using single-channel EOG. Discrete Wavelet Transform (DWT) was used to evaluate single channel EOG signals using different statistical properties. As a result of the study, it has been determined that single channel EOG performs better than single channel EEG in sleep classification studies [25].

The EOG measuring system was designed, and the classification of the horizontal and vertical motions of the eyeball was given for human computer interfaces that can be regulated by eye movements, in a study on the classification of electrooculogram signals and determination of direction of eye movements. [26].

A new intelligent sensing model was proposed in a study that used an artificial neural network to assess the direction of eye movements based on electrooculogram signals. In addition to traditional eye movement tracking methods, the model includes tick detection and blinking. The properties of EOG signals are extracted and fed into a feed forward artificial neural network. According to the results, the proposed model may be a useful method for detecting eye movements [27].

A mobile game called "EndeavorRx" has been developed, and its efficacy has been tested in the treatment of ADHD. This mobile game, which was created to be used as a medication, has also earned FDA approval. Studies on applications that can be used in computer and mobile settings for ADHD diagnosis, follow-up, and treatment have gained traction in recent years as technology has become more integrated into our lives [28].

As can be seen from the current studies above, EEG signals were generally analyzed for detection of ADHD. In this study a novel computer-based ADHD diagnostic support and 
follow-up system is proposed with eye tracking approach using EOG signals. It is analyzed different parameters from previous work of our team [29-30].

There are studies in the literature on the tracking of eye movements using a video camera. However, these systems (eyetracking devices) cannot be as effective as the sensitivity of an eye movement detecting systems obtained from physiological signals, and the systems that can provide precise video recording are high cost and will spend more processing load due to the image processing studies. Therefore, eye tracking system is not preferred.

In this study, EOG based follow up and diagnosis of ADHD decision support system is proposed. For this aim a test system is developed and performance parameters are obtained as features. Artificial Neural Networks (ANN) are applied for classification of ADHD and healthy group using obtained features.

The rest of the paper is organized as follows. Section 2 gives information about Materials and Methods. Section 3 describes the obtained results. Finally, Section 4 concludes the paper.

\section{MATERIAL AND METHOD}

Ethics Committee Approval required for our study was obtained from Erciyes University Faculty of Medicine before the study. Children and adolescents with ADHD between the ages of 8-13, both genders, right-handed, were included in the study. The control group was made up of people who were similar to the study group in terms of age and gender. No identified neurological, metabolic, endocrinological, or psychological conditions, boys and girls in the "8-13" age range, IQ over 85, and no hearing or vision problems were among the requirements for control group volunteers in the study.

The DSM-V diagnostic criteria for ADHD, being in the " 8-13 " age group, not using disease-related drugs, having an IQ above 85, and no hearing or vision disorders are the inclusion criteria for ADHD. Individuals who did not meet the above-mentioned inclusion criteria were not allowed to participate.

The diagnosis of ADHD patients was made by the Department of Child and Adolescent Mental Health, Erciyes University. All IQ measurements were made with the WISC-R test. The follow-up of the response to treatment was done with the Conners Rating Scale-Parent Teacher Form and the Clinical Global Follow-up Evaluation Scale.

The materials and methods of this study is similar to previous study of our research team as seen in ref [30]. 


\subsection{Description of Visual Stimulus Follow-up Test}

The developed visual stimulus follow-up test offers the opportunity to examine horizontal and vertical eye movements under visual and auditory stimuli. Thus, the change in the attention state of the person whose EOG measurement was performed under various visual and auditory distractors can be evaluated. Visual stimulus tracking test was prepared in C \# programming language. A 24 " monitor was used in the study. At a distance of $30 \mathrm{~cm}$ from the screen, the test was applied so that the eye level of the individual was at the same level as the midpoint of the screen.

The test software starts with the tracking of white visual stimuli on a black background after calibration and continues with the determination of the visual stimulus in 10 different colors of the background and the previous background color as seen in Figure 1. The test consists of 3 stages. In the first stage, only visual stimuli act as "square" and "z". The same test is repeated for 10 periods and takes 55 seconds in total.

Following that, the test's second and third stages begin. Visual distractors were applied to the test in the second step, in addition to the test in the first. Pictures of cats and dogs appear at a different time than the stimulus. The test's second stage lasts 60 seconds. The third stage of the test starts after that. The third section of the test looks just like the second. Auditory distractors are also included in the third section. The third and final portion of the test lasts 60 seconds. Below is a visual representation of the material of the developed visual stimulus follow-up test in general. While the participants watch the screenshots revealed during the evaluation, EOG records are taken simultaneously. The testing process was carried out for approximately 3 minutes.

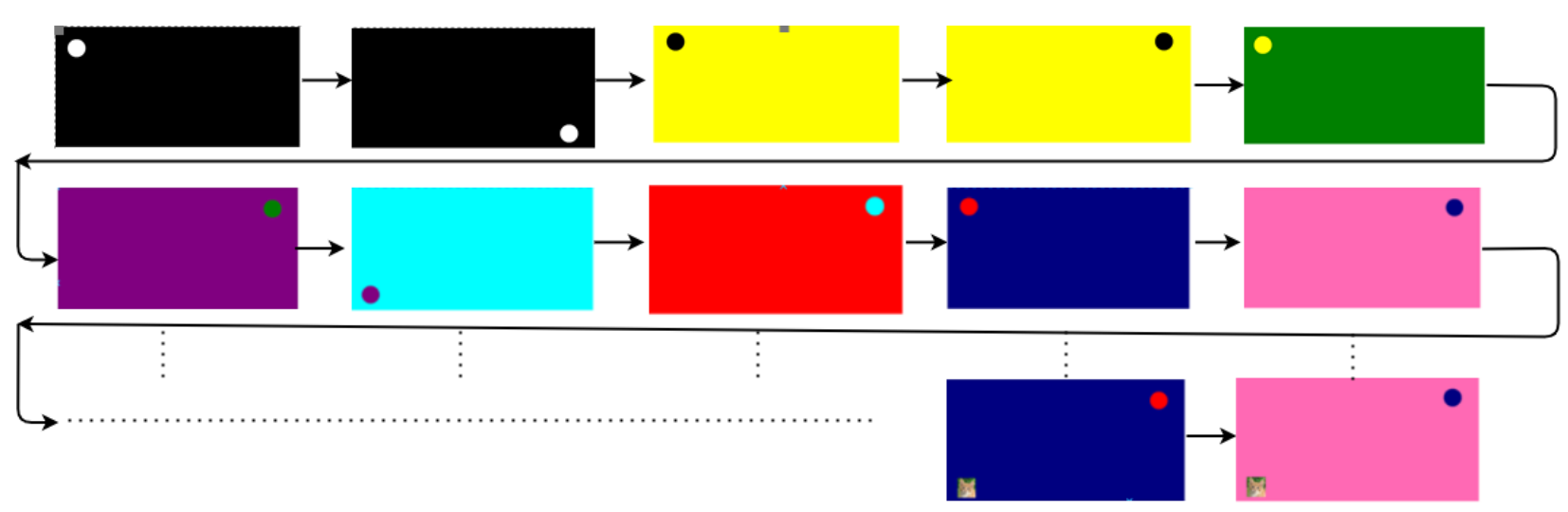

Figure 1. Visual Stimulus Follow-up Test 


\subsection{Recording of The EOG Signals}

To obtain EOG signals, EOG recordings were performed using the BIOPAC-MP150 recorder manufactured by Biopac Systems Inc. and two EOG100C electrooculogram modules connected to the main device [31]. The electrical potential difference of non-invasive surface electrodes connected to the right, left, upper, and lower regions of the eye relative to the reference electrode was used to obtain EOG signals. Surface electrodes with a non-invasive $\mathrm{Ag}-\mathrm{AgCl}$ water-based adhesive were used. The recording system was used to collect signals from patients who had been diagnosed with ADHD for the first time. Later, after 6 months of drug therapy, second records were performed. Thus, the evaluation of the effectiveness of drug therapy was also provided using proposed approach. Apart from this group, EOG signals were also recorded from healthy individuals, and evaluated as a control group in the study. All EOG signals were recorded in two channels, and analyzed in MATLAB.

\subsection{Processing of The EOG Signals}

EOG signals are a non-stationary signal. Therefore, a variety of attributes are needed to extract useful information from the signal. The features used in our study were calculated from the EOG signals obtained as a result of the visual stimulus follow-up test applied to patients and healthy individuals.

The study is performed with calculating of four attribute parameters, that are the total number of movements parameter, the visual distractor follow-up parameter, the visual stimulus movement parameter, and the audible stimulus identification parameter, in order to distinguish between patient and healthy people.

The following preprocessing methods were applied to the EOG signals before the defined attributes were calculated.

For denoising of the EOG signal, Singular Spectrum Analysis (SSA) method [32, 33] is applied to the EOG signals. As a result, the application of the SSA method to the EOG signals, these signals are denoised and transformed into a form suitable for detecting eye movements.

The EOG signals were recorded at a sampling frequency of $500 \mathrm{~Hz}$. First of all, the sampling frequency has been reduced to $20 \mathrm{~Hz}$ to reduce the processing load. Then, the amplitude normalization process was performed by assigning EOG signal between 0-1 amplitude values. 
The normalized EOG signals were divided into four sections: right and left eye movements in horizontal eye movements, upward and downward eye movements in vertical eye movements. Eye movements in each direction were evaluated in all 3 parts of the visual stimulus follow-up test (no distractor, visual distractor, visual + auditory distractor).

The number of eye movements and direction were used to obtain reference data in the visual stimulation follow-up examination. The details about what time, how many times to look in which direction, and when and where distractors appeared was calculated using the reference data. The EOG signals recorded from patient and healthy subjects were compared to reference data. An example of the recorded and deoised EOG signals using SSA technique is seen in Figure $2 a$ and $2 b$.

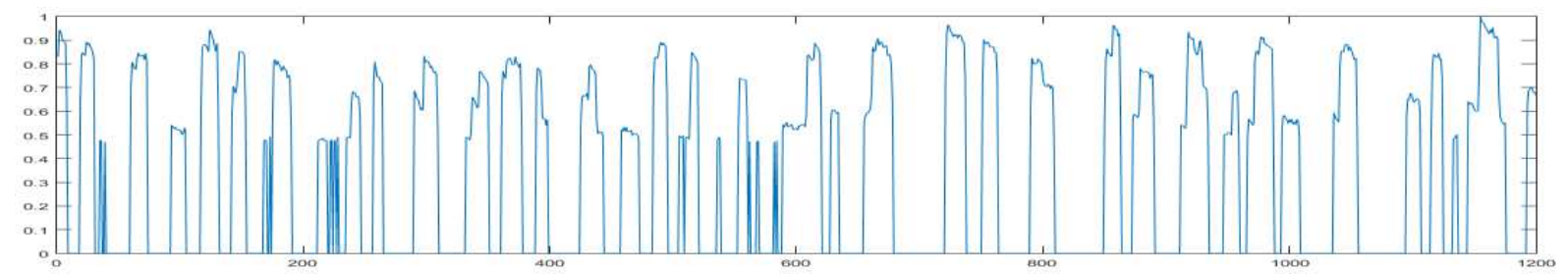

Figure 2a. An example of The EOG signals (belong to right eye movement with Visual

Distractor)

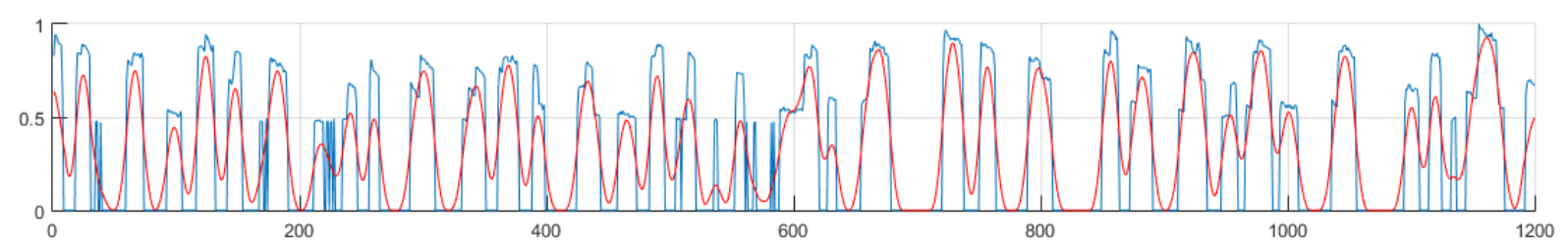

Figure 2b. Application of SSA to the EOG signals

All the recorded data were processed in MATLAB environment and evaluations were carried out using the following 4 parameters. Analysis results are presented in the results section.

\subsection{Calculated Features}

In this study, the first record EOG signals were obtained from 64 patients diagnosed with ADHD according to DSM criteria, with a visual stimulus follow-up test.

11 of the EOG signals consist of individuals using drugs and 53 of the EOG signals are the first records. Second records were performed from the patients after drug treatment. In the second record, the number of people participating in this study is 39 .

The total number of EOG signals recorded from the control group diagnosed with ADHD according to the DSM criteria is 36 . This study was performed using the total number of 128 
EOG signals, including 53 patients' data recorded first time, 39 patients' second data recorded after drug treatment, and 36 healthy subjects' data.

\section{The Parameters Analyzed in this Study are defined below;}

1- Total Number of Movements (TM): In this parameter, the total number of eye movements separated by directions of healthy and ADHD individuals was compared with the total number of reference movements. The reference movement number is the number obtained as a result of determining how many movements the eye moves in which direction in the visual stimulus follow-up test. The following process steps for the TM parameter were obtained in the Matlab environment.

Step-1 In the visual stimulus follow-up test, identify how many times all visual stimuli appear in which direction.

Step-2 Determine in which direction and how many times each eye movement occurred from the EOG signals of the individual on whom the visual stimulus follow-up test was applied.

Step-3 With the reference value, calculate the proportionality of the direction (right, left, up, down) and number details (number of eye movements) obtained in Step-2.

Step-4 Repeat steps 2 and 3 for all patient / healthy records and determines the total number of movements and success rates for all individuals.

2- Number of Visual Distractor Follow Up (VDF): In the second (visual stimulus) and third (visual + auditory) stage of the visual stimulus follow-up test, the images of cats and dogs that appear on the screen are used as a distractor, and during the test, it is ensured that the eye is looking at that area at the location and time these distractors appear on the screen. The desired situation is not to look at the distractors and the eye movements should not be in the direction of the distractors. The following process steps for the VDF parameter were obtained in the Matlab environment.

Step-1 In the visual stimulus follow-up test, identify at what time and in what position the "cat" and "dog" distractors appear on the screen.

Step-2 Determine which location the eye is looking at when the "cat" and "dog" appear on the screen using the signals of the subject to whom the visual stimulus follow-up test is applied.

Step-3 Determine whether the time and location information obtained in Step-2 is compatible with the time and location information obtained in Step-1, and calculates the correct number of follow-ups in the event of visual distraction. 
Step-4 Repeat steps 2 and 3 for all EOG signals recorded from patient / healthy subjects and calculate correct follow-up numbers in case of all visual distractions.

3- Detection of Visual Stimulus (VSD): In the visual stimulus follow-up test, the stimuli appear on the screen periodically in the directions of the "square" and "z" shape, and the individuals on whom the test was applied were asked whether they could detect the visual stimulus movement, that is, whether they could perceive the square and $\mathrm{z}$ state, and the evaluation was made according to their answers. The practitioner then asks the patient / healthy subjects how the visual stimulus action occurs at the end of the test. This parameter is evaluated as "successful" if "square" and "z" movement can be described, and "failed" in the incomplete description.

4- Detection of Audible Stimulus (ASD): There are audible stimuli in the third stage of the visual stimulus follow-up test. These stimuli consist of the ambulance alarm sound and the sounds of children at school. The individuals on whom the test was applied were asked what sound they heard. The evaluation was made according to their answers.

The practitioner then asks the patient / healthy subjects what sound the auditory stimulus is and what he heard at the end of the test. This parameter is evaluated as "successful" if "child sound" and "ambulance / alarm sound" can be described, and "unsuccessful" in an incomplete description.

\subsection{Artificial neural networks}

Artificial neural networks are a type of information processing system that is influenced by biological neural networks and shares some of their performance characteristics. Recent ANN research has revealed that ANN has strong pattern classification and pattern recognition capabilities, and that it is commonly used in data estimation due to advantages such as the ability to manage nonlinear structures and parallel and serial processing capacity. The sum and activation functions of the processor elements, the learning strategy, the learning rule used, and the topology as a result of the interaction of process elements decide the model of the network in an ANN structure [34,35]. The ANN is formed when artificial neural cells (neurons) come together. Neurons aren't collected at random. Cells, in general, form a threelayer network in which they are arranged in parallel in each layer. The multi-layer perceptron (MLP) model, which consists of an input layer, one or more hidden layers, and an output layer, is the most commonly used version of ANN. The input layer's processor elements function as a buffer, distributing input signals to the hidden layer's processing elements. 
Each of these components adds the input data to the sum function by multiplying it by the weight coefficients, which display the efficiency of the input over the hidden neuron. Following that, these sum functions are passed through a transfer function, and the neuron's output value is determined as follows:

$y_{j}=f\left(\sum_{i} w_{i j} a_{i}+\theta\right)$

Where $\mathrm{j}$ denotes the number of neurons, I denotes the number of inputs, input signal, weight coefficient, and is the bias expression (or threshold).

Many of the processors in this layer perform the same operations. The network output values are determined using the processor elements in the output layer as hidden layer elements. The output of neurons in the output layer is computed in a similar way. The number of squared differences between the desired and actual values of the output neurons $\mathrm{E}$ can be calculated using the equation below:

$E(w)=\sum\left(y_{d j}-y_{i}\right)^{2}$

Where $y_{d j}$ is the target (actual) output value.

The number of input parameters and hidden neurons in an ANN model have a major effect on the model's classification efficiency.

From one to twelve numbers of neurons in the hidden layer were set in the architecture of the ANN in this study using the 'for' loop in Matlab software, and the optimum architecture of the model was calculated based on the giving minimum mean square error for classification of training performance. In order to prevent overfitting, it is also important to determine the number of hidden layers. As a result, the number of hidden layers in this study is set to one to prevent overfitting.

In the MLP model, information flows in the forward direction. The MLP model is also known as feed forward ANN because of this feature. One of the benefits of this model is that it can train the network using a variety of learning algorithms. The weights of the network are adjusted according to the training algorithm until the error between the network's output and the target output is reduced. Back Propagation Neural Network is the name given to the MLP model when it is supervised by a learning algorithm (BPNN). The feed forward network, or 
BPNN, is the most widely used ANN model in time series estimation. The BPNN feed forward network structure was used in our study.

Since the network's job is to generate an output for each input, MLP-ANN is based on a supervised learning strategy. There are two phases to MLP-ANN learning. The output of the network is computed first, in the forward calculation step. The weights are determined in the second stage, the backward calculation stage, based on the difference between the estimated output and the output of the networks.

MLP-learning ANN's rule is known as the back-propagated MLP learning rule since it is implemented in this manner. Different learning algorithms are used to train the network in the ANN. For the classification of ADHD and control group, the Levenberg Marquardt (LM) learning algorithm is used, which has a computational speed advantage over the ANN [36].

\section{3- RESULTS}

The first record EOG signals were obtained using the proposed method from patients who were diagnosed with ADHD in the clinic but did not applied any drug treatment. Following that, drug therapy was applied to these patients, and after 6 months of treatment, new EOG signals were recorded as the second record EOG signals. In addition, the EOG signals were recorded from healthy individuals as the control group. The obtained attributes are shown in Table 1-4. The numbers 1, 2, 3 (as an example left-1, right-2) indicates the parts of the visual stimulus follow-up test; 1 : the non-distractor part, 2: the visual distractor part, 3: the visual + auditory distractor part in the tables.

Eye movements were detected using EOG signals obtained from patients (before and after treatment) and healthy individuals, and for three parts of the visual stimulus follow-up test (without distractors, with visual distractors, with visual + auditory distractors), in each direction (left, right, up, down) eye movement numbers were calculated. Table 1 shows the success ratio with the number of eye movements and reference movements (which should be according to the visual stimulus follow-up test). 
Table 1. Averages of Total Number of Movements by Direction and Ratios with Reference Movement

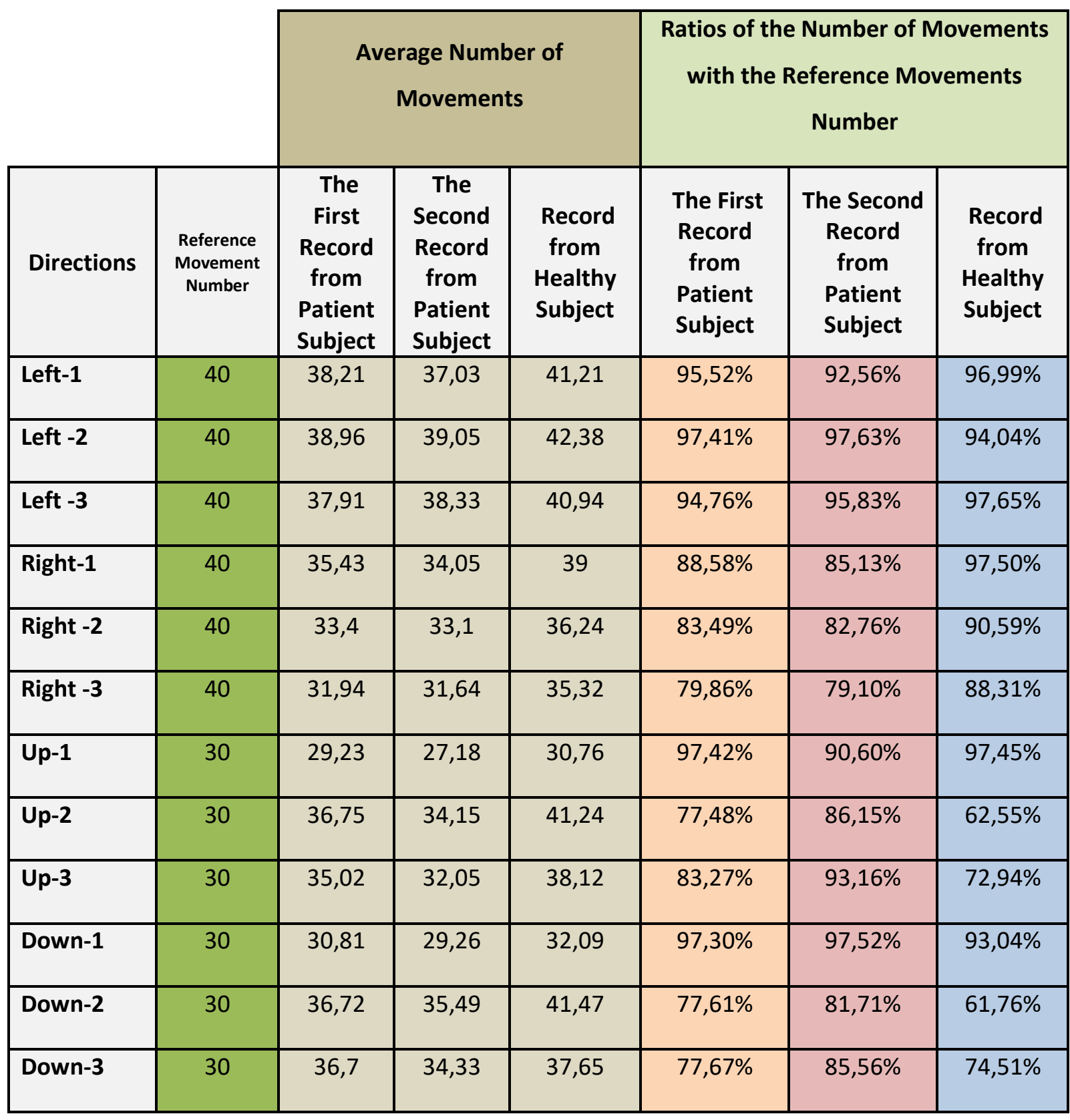

The number of movements were determined separately in three parts of the visual stimulus follow-up test, and the success ratio of the patients and all healthy participants were calculated according to the directions and presented in Table 2. 
Table 2. Ratio of Success for Number of Visual Distractor Follow Up

\begin{tabular}{|c|c|c|c|c|c|c|c|c|c|}
\hline \multirow[b]{2}{*}{ Directions } & \multicolumn{3}{|c|}{ TEST WITH NON DISTRACTORS } & \multicolumn{3}{|c|}{$\begin{array}{l}\text { TEST WITH VISUAL } \\
\text { DISTRACTORS }\end{array}$} & \multicolumn{3}{|c|}{$\begin{array}{c}\text { TEST WITH VISUAL AND AUDIBLE } \\
\text { DISTRACTORS }\end{array}$} \\
\hline & $\begin{array}{l}\text { The } \\
\text { First } \\
\text { Record } \\
\text { from } \\
\text { Patient } \\
\text { Subject }\end{array}$ & $\begin{array}{l}\text { The } \\
\text { Second } \\
\text { Record } \\
\text { from } \\
\text { Patient } \\
\text { Subject }\end{array}$ & $\begin{array}{c}\text { Record } \\
\text { from } \\
\text { Healthy } \\
\text { Subject }\end{array}$ & $\begin{array}{c}\text { The } \\
\text { First } \\
\text { Record } \\
\text { from } \\
\text { Patient } \\
\text { Subject }\end{array}$ & $\begin{array}{l}\text { The } \\
\text { Second } \\
\text { Record } \\
\text { from } \\
\text { Patient } \\
\text { Subject }\end{array}$ & $\begin{array}{l}\text { Record } \\
\text { from } \\
\text { Healthy } \\
\text { Subject }\end{array}$ & $\begin{array}{c}\text { The } \\
\text { First } \\
\text { Record } \\
\text { from } \\
\text { Patient } \\
\text { Subject }\end{array}$ & $\begin{array}{l}\text { The } \\
\text { Second } \\
\text { Record } \\
\text { from } \\
\text { Patient } \\
\text { Subject }\end{array}$ & $\begin{array}{c}\text { Record } \\
\text { from } \\
\text { Healthy } \\
\text { Subject }\end{array}$ \\
\hline Left & $95,52 \%$ & $92,56 \%$ & $96,99 \%$ & $97,41 \%$ & $97,63 \%$ & $94,04 \%$ & $94,76 \%$ & $95,83 \%$ & $97,65 \%$ \\
\hline Right & $88,58 \%$ & $85,13 \%$ & $97,50 \%$ & $83,49 \%$ & $82,76 \%$ & $90,59 \%$ & $79,86 \%$ & $79,10 \%$ & $88,31 \%$ \\
\hline Up & $97,42 \%$ & $90,60 \%$ & $97,45 \%$ & $77,48 \%$ & $86,15 \%$ & $62,55 \%$ & $83,27 \%$ & $93,16 \%$ & $72,94 \%$ \\
\hline Down & $97,30 \%$ & $97,52 \%$ & $93,04 \%$ & $77,61 \%$ & $81,71 \%$ & $61,76 \%$ & $77,67 \%$ & $85,56 \%$ & $74,51 \%$ \\
\hline Mean & $94,71 \%$ & $91,45 \%$ & $96,24 \%$ & $84,00 \%$ & $87,06 \%$ & $77,24 \%$ & $83,89 \%$ & $88,41 \%$ & $83,35 \%$ \\
\hline
\end{tabular}

The test applied during the recording of EOG signals was made on 16: 9 size monitors. Due to these screen sizes, horizontal eye movements were detected more effectively, while vertical eye movements were detected with smaller amplitudes. There were also cases where vertical eye movements were largely undetectable during some measurements. In this context, horizontal eye movements are considered to be more decisive in the determination of the patient / healthy subjects.

While evaluating the following performance of visual distraction, it was determined whether the patients and all healthy individuals followed the "cat" and "dog" pictures on the screen in the visual stimulus follow-up test. For this, the position of the eye when the distracting image appears on the screen has been determined and compared with the position of the distractor. Thus, it was determined whether the distractor was looked at or not. The desired situation is that individuals are not affected by the distractor and is that individuals continue follow-up tests. Based on the reference movement number, the following performance of the test was determined and the success ratio was calculated. There are visual distractors in the 2nd (visual distractor) and 3rd (visual + auditory distractor) sections of the visual stimulus follow-up test, and the success ratio in these sections are determined and presented in Table 3. 
Table 3. Performance of Visual Distractor Tracking Test

\begin{tabular}{|l|c|c|c|c|c|c|}
\cline { 2 - 7 } \multicolumn{1}{c|}{} & \multicolumn{3}{|c|}{ TEST WITH VISUAL } & \multicolumn{2}{c|}{ TEST WITH VISUAL AND AUDIBLE } \\
\hline \multirow{2}{*}{ DISTRACTORS } & $\begin{array}{c}\text { The } \\
\text { First } \\
\text { Record } \\
\text { from } \\
\text { Datient } \\
\text { Subject }\end{array}$ & $\begin{array}{c}\text { The } \\
\text { Second } \\
\text { Record } \\
\text { from } \\
\text { Patient } \\
\text { Subject }\end{array}$ & $\begin{array}{c}\text { Record } \\
\text { from } \\
\text { Healthy } \\
\text { Subject }\end{array}$ & $\begin{array}{c}\text { The First } \\
\text { Record } \\
\text { from } \\
\text { Patient } \\
\text { Subject }\end{array}$ & $\begin{array}{c}\text { The } \\
\text { Second } \\
\text { Record } \\
\text { from } \\
\text { Patient } \\
\text { Subject }\end{array}$ & $\begin{array}{c}\text { Record } \\
\text { from } \\
\text { Healthy } \\
\text { Subject }\end{array}$ \\
\hline Left-Cat & $65,66 \%$ & $66,15 \%$ & $67,78 \%$ & $71,51 \%$ & $68,21 \%$ & $65,28 \%$ \\
\hline Right-Dog & $75,66 \%$ & $78,21 \%$ & $82,22 \%$ & $73,40 \%$ & $68,46 \%$ & $71,11 \%$ \\
\hline Mean & $70,66 \%$ & $72,18 \%$ & $75,00 \%$ & $72,45 \%$ & $68,33 \%$ & $68,19 \%$ \\
\hline
\end{tabular}

The stimuli appear on the screen regularly in the directions of the "square" and "z" form in the visual stimulus follow-up test, and the individuals on whom the test was applied were asked if they could detect the visual stimulus movement, that is, if they could perceive the square and $\mathrm{z}$ pattern, and the evaluation was made based on their responses. Table 4 shows the performance of visual stimulus detection.

Table 4. Performance of Visual Stimulus Detection

\begin{tabular}{|c|c|c|}
\hline Healthy Subject & $\begin{array}{c}\text { The First Record from Patient } \\
\text { Subject }\end{array}$ & $\begin{array}{c}\text { The Second Record from } \\
\text { Patient Subject }\end{array}$ \\
\hline $\mathbf{7 5 , 6 1 \%}$ & $\mathbf{3 3 , 8 5 \%}$ & $\mathbf{5 0 , 0 0 \%}$ \\
\hline
\end{tabular}

In the third stage of the visual stimulation follow-up evaluation, there are audible stimuli. The sound of an ambulance alarm and the sounds of children at school are among the triggers. The individual who were subjected to the test were asked what sound they heard. Their answers were used to determine their performance as seen in Table 5.

Table 5. Performance of Audible Stimulus Detection

\begin{tabular}{|c|c|c|}
\hline Healthy Subject & $\begin{array}{c}\text { The First Record from Patient } \\
\text { Subject }\end{array}$ & $\begin{array}{c}\text { The Second Record } \\
\text { from Patient Subject }\end{array}$ \\
\hline $90,24 \%$ & $63,07 \%$ & $60,00 \%$ \\
\hline
\end{tabular}

If the obtained results are evaluated, some performance parameters are distinctive for ADHD (before, after treatment) and healthy subjects. It is seen from tables that, Right-1, Right-2, 
Right-3 features from TM parameter and Dog Right-2 feature from VDF parameter are distinctive for a patient and healthy group.

The VSD and ASD parameters have a high success rate in detecting the patient/healthy group. The classification analysis was performed using parameters that are Right-1, Right-2, Right-3 features from TM parameter and Dog Right-2 feature from VDF parameter as well as VSD and ASD parameters. In the study, 3 group classification procedures were performed: patient1 (before drug treatment), patient-2 (after drug treatment), and healthy. The training set consisted of $70 \%$ of the data, while the test sets consisted of the remaining $30 \%$ of the data. Six inputs have been applied to the ANN and there is one target output. The performance of the ANN is seen in Table 6.

Table 6. Artificial Neural Network Classification Success

\begin{tabular}{|c|c|c|}
\hline $\begin{array}{c}\text { Test performance for Healthy } \\
\text { Subjects }\end{array}$ & $\begin{array}{c}\text { Test performance for ADHD } \\
\text { Subjects (non-treated) }\end{array}$ & $\begin{array}{c}\text { Test performance for Healthy } \\
\text { Subjects (after treatment) }\end{array}$ \\
\hline $81,76 \%$ & $62,54 \%$ & $75,78 \%$ \\
\hline
\end{tabular}

\section{5- CONCLUSION AND DISCUSSION}

When the TM, VDF, VSD, and ASD parameters were compared for patients and healthy people, it was found that healthy people had a higher success rate than ADHD individuals.

In addition, when obtained result for individuals receiving drug treatment is analyzed, it is clearly seen that test performance increase significantly and test performance is observed even at higher ratio than healthy individuals in some parameters (as example up-direction feature of TM parameter). This suggests that drug therapy improves the test performance. According to the result obtained from ANN, the classification performance was found to be high in healthy and drug-treated patients, and low in non-treated patients.

In this context, our studies continue and it is aimed to carry the existing study forward by increasing the existing parameters, developing analysis techniques, increasing the number of measurements, developing the artificial neural network application. We anticipate that this study, which was developed as an auxiliary system for ADHD diagnosis, will contribute to the diagnosis of the disease with high accuracy rates in the future. Our studies continue to develop auxiliary parameters and analysis procedures that can be used in the diagnosis of ADHD using the EOG records obtained. Findings obtained from the current study show promising results that can be used in the detection of ADHD. 


\section{Compliance with Ethical Standards}

Conflict of Interest The authors declare that they have no conflict of interest.

Ethical Approval All procedures performed in studies involving human participants were in accordance with the ethical standards of the institutional and/or national research committee and with the 1964 Helsinki declaration and its later amendments or comparable ethical standards.

Informed Consent Informed consent was obtained from all individual participants included in the study.

\section{ACKNOWLEDGEMENT}

This study is supported by the Scientific and Technological Research Council of Turkey (TUBITAK) with 116E614 project number. We thank TUBITAK for their support. 


\section{REFERENCES}

[1] Barkley, R.A. 2006. Attention-deficit hyperactivity disorder: A handbook for diagnosis and treatment (3. Basim ). New York: Guilford Press.

[2] Spencer, T.J., Biederman, J., Mick, E. 2007. "Attention deficit/hyperactivity disorder: Diagnosis, lifespan, comorbidities, and neurobiology", Ambulatory Pediatrics, 7,73-81.

[3] American Psychiatric Association. 2000. Diagnostic and Statistical Manual of Mental Disorders (4. Basım) Text Revision (DSM-IV-TR). Washington DC.

[4] Nigg, J.T. 2000. "On inhibition/disinhibition in developmental psychopathology: Views from cognitive and personality psychology and a working inhibition taxonomy", Psychological Bulletin, 126,220-246.

[5] Biederman, J., Monuteaux, M.C., Mick E., Spencer, T., Wilens, T.E., Silva, J.M. 2006. "Young adult outcome of attention deficit hyperactivity disorder: a controlled 10yearfollow-upstudy", PsychologicalMedicine,36,167-179.

[6] Nolan, E.E., Gadow, K.E., Sprafkin J.J. 2001. “Teacher reports of DSM-IV ADHD, ODD, and CD symptoms in schoolchildren”, J Am Acad Child Adolesc Psychiatry, 40, 241-9.

[7] Gaub, M., Carlson, CL. 1997. “ Gender differences in ADHD: a metaanalysis and critical review”, J Am Acad Child Adolesc Psychiatry, 36, 1036-1045.

[8] Montiel-Nova, C. 2003. "Epidemiological data about attention deficit hyperactivity disorder in a sample of Morabina Children”, Rev Neurol, 37, 815-823.

[9] Motavallı, N. 1994. "Kentsel kesimde Türk ilkokul çocuklarında Dikkat Eksikliği Hiperaktivite Bozukluğunun Nozolojik bağlamda prevalansının araştırılması”, Uzmanlık tezi, İstanbul üniversitesi tıp fakültesi, İstanbul.

[10] Conners, C. K., Sitarenios, G., Parker, J. D. A., Epstein, J. N. 1998. "Revision and restandardization of the Conners Teacher Rating Scale (CTRS-R): Factor structure, reliability and criterion validity”, Journal of Abnormal Child Psychology, 26 (4), 279-291.

[11] Guy, W. 1976. Clinical Global Impressions: In ECDEU Assessment Manual for Psychopharmacology, Sayfa 218-222. Revised DHEWPub. (ADM).Rockville, MD:National Institute for Mental Health. 
[12] Conners, C. K., Sitarenios, G., Parker, J. D. A., Epstein, J. N. 1998. "The Revised Conners Parent Rating Scale (CPRS-R): Factor structure, reliability, and criterion validity", Journal of Abnormal Child Psychology, 26 (4), 257-268.

[13] Benjamin, K. 1986. "Spectral analysis and discrimination by zero-crossings", Proceedings of the IEEE , 74(11), 1477-1493.

[14] Yazgan, E., Korürek, M., 1996. T1p Elektroniği. İstanbul: İTÜ

[15] Wall, R.W. 2003. "Simple methods for detecting zero crossing" , Industrial Electronics Society, 3, $2477-2481$.

[16] Liu, C.C., Chaovalitwongse, W.A., Pardalos, P.M., Seref, O., Xanthopoulos, P., Sackellares, J.C., Skidmore, F.M., 2007. Quantitative analysis on electrooculography (EOG) for neurodegenerative disease, pp 247-251. Systems Analysis and Optimization in Biomedicine, March 28-30, 2007, Gainesville.

[17] Ilona Merikanto, Liisa Kuula, Tommi Makkonen, Risto Halonen, Jari Lahti, Kati Heinonen, Katri Räikkönen, Anu-Katriina Pesonen,ADHD symptoms are associated with decreased activity of fast sleep spindles and poorer procedural overnight learning during adolescence, Neurobiology of Learning and Memory, Volume 157,2019,Pages 106-113.

[18] Juri D. Kropotov, Marina V. Pronina, Valery A. Ponomarev, Yury I. Poliakov, Irina V. Plotnikova, Andreas Mueller,Latent ERP components of cognitive dysfunctions in ADHD and schizophrenia, Clinical Neurophysiology, Volume 130, Issue 4,2019,Pages 445-453.

[19] Chien-Lin Yu, Ting-Yu Chueh, Shu-Shih Hsieh, Yu-Jung Tsai, Chiao-Ling Hung, Chung-Ju Huang, Chien-Ting $\mathrm{Wu}$, Tsung-Min Hung, Motor competence moderates relationship between moderate to vigorous physical activity and resting EEG in children with ADHD, Mental Health and Physical Activity,Volume 17,2019,100302.

[20] Juan Lopez Marcano, Martha Ann Bell, A.A. (Louis) Beex, Classification of ADHD and non-ADHD subjects using a universal background model, Biomedical Signal Processing and Control, Volume 39,2018,Pages 204-212.

[21] Hüseyin Öztoprak, Mehmet Toycan, Yaşar Kemal Alp, Orhan Arıkan, Elvin Doğutepe, Sirel Karakaş, Machine-based classification of ADHD and nonADHD participants using time/frequency features of event-related neuroelectric activity,Clinical Neurophysiology, Volume 128, Issue 12, 2017,Pages 2400-2410. 
[22] Julián J. González, Leopoldo D. Méndez, Soledad Mañas, Mª Rosario Duque, Ernesto Pereda, Luis De Vera, Performance analysis of univariate and multivariate EEG measurements in the diagnosis of ADHD, Clinical Neurophysiology, Volume 124, Issue 6, 2013, Pages 1139-1150.

[23] Daniel F. Hermens, Michael R. Kohn, Simon D. Clarke, Evian Gordon, Leanne M. Williams, Sex differences in adolescent ADHD: findings from concurrent EEG and EDA, Clinical Neurophysiology, Volume 116, Issue 6, 2005, Pages 1455-1463.

[24] Hideyuki Kanematsu, Dana M. Barry, Nobuyuki Ogawa, Katsuko T. Nakahira, Michiko Yoshitake, Tatsuya Shirai, Masashi Kawaguchi, Toshiro Kobayashi, Kuniaki Yajima, Some Psychological Responses Measured by a Commercial Electrooculography Sensor and Its Applicability, Procedia Computer Science, Volume 126, 2018, Pages 10141022.

[25] Md Mosheyur Rahman, Mohammed Imamul Hassan Bhuiyan, Ahnaf Rashik Hassan, Sleep stage classification using single-channel EOG, Computers in Biology and Medicine,Volume 102, 2018, Pages 211-220.

[26] Anwesha Banerjee, Shreyasi Datta, Monalisa Pal, Amit Konar, D.N. Tibarewala, R. Janarthanan, Classifying Electrooculogram to Detect Directional Eye Movements, Procedia Technology, Volume 10, 2013, Pages 67-75.

[27] Hande Erkaymaz, Mahmut Ozer, İlhami Muharrem Orak, Detection of directional eye movements based on the electrooculogram signals through an artificial neural network, Chaos, Solitons \& Fractals, Volume 77, 2015, Pages 225-229.

[28] Kollins, Scott $\mathrm{H}$ et al., A novel digital intervention for actively reducing severity of paediatric ADHD (STARS-ADHD): a randomised controlled trial, The Lancet Digital Health, Volume 2, Issue 4, 168 - 178, 2020.

[29] Latifoğlu, F., Esas, M.Y., Demirci, E., Diagnosis of attention-deficit hyperactivity disorder using EOG signals: a new approach, Biomedical Engineering / Biomedizinische Technik, Volume 65, Issue 2, Pages 149-164, eISSN 1862-278X, ISSN 00135585, DOI: https://doi.org/10.1515/bmt-2019-0027.

[30] Latifoğlu, F., Esas, M.Y., İleri, R., Taştepe, N., Özmen, S., Demirci, E., Evaluation of Attention Deficit Hyperactivity Disorder With A Novel Approach Using an EOG Based Visual Stimulus Tracking Test, 2020 (yayınlanma aşamasında). 
[31] https://www.biopac.com/wp-content/uploads/MP150-Systems.pdf (Erişim Tarihi: Nisan 2019)

[32] Hassani, H. 2007. "Singular spectrum analysis: methodology and comparison", Journal of Data Science, 5, 239-257.

[33] Mert, C.,Milnikov A. 2011."Singular spectrum analysis method as auniversal fitler", 1-5. Application of Information and Communication Technologies

[34] Hagan M.T., Demuth H.B., Beale M.H., 1996, "Neural Network Design. Boston. MA: PWS Publishing.

[35] Haykin S., 1994, "Neural Networks: A Comprehensive Foundation", Macmillan College Publishing Company Inc., New.

[36] Marquardt DW., 1963, “An algorithm for least-squares estimation of nonlinear parameters", Journal of the society for Industrial and Applied Mathematics. 11: 431-441. 
Figures

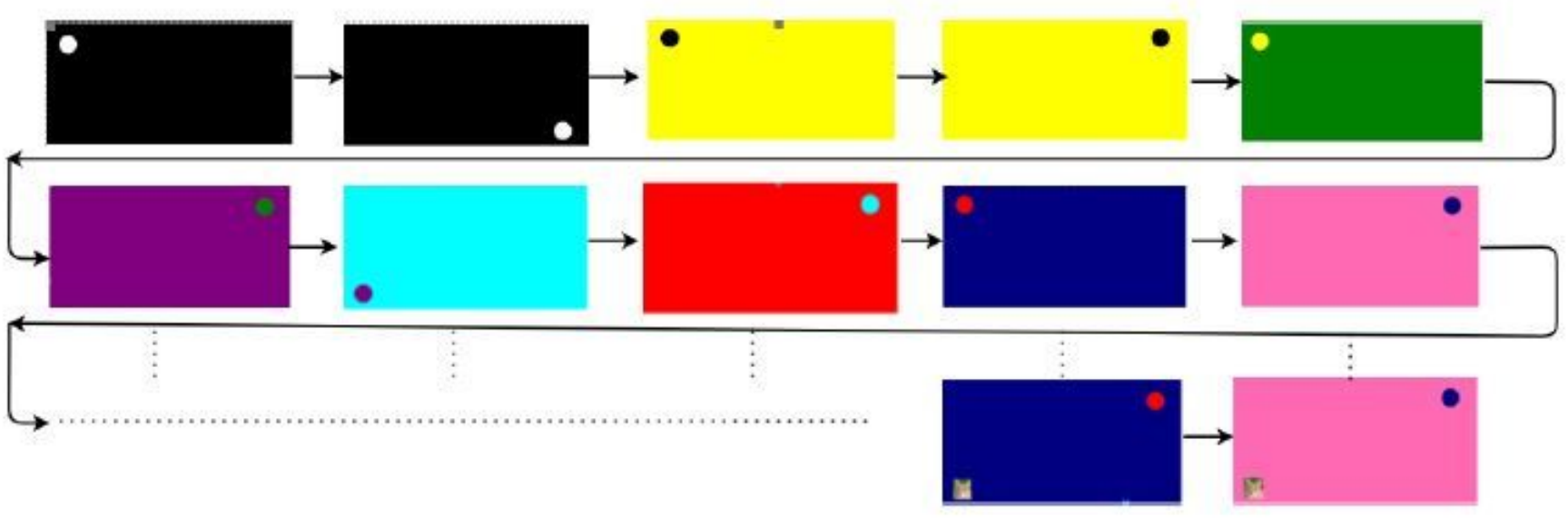

Figure 1

Visual Stimulus Follow-up Test
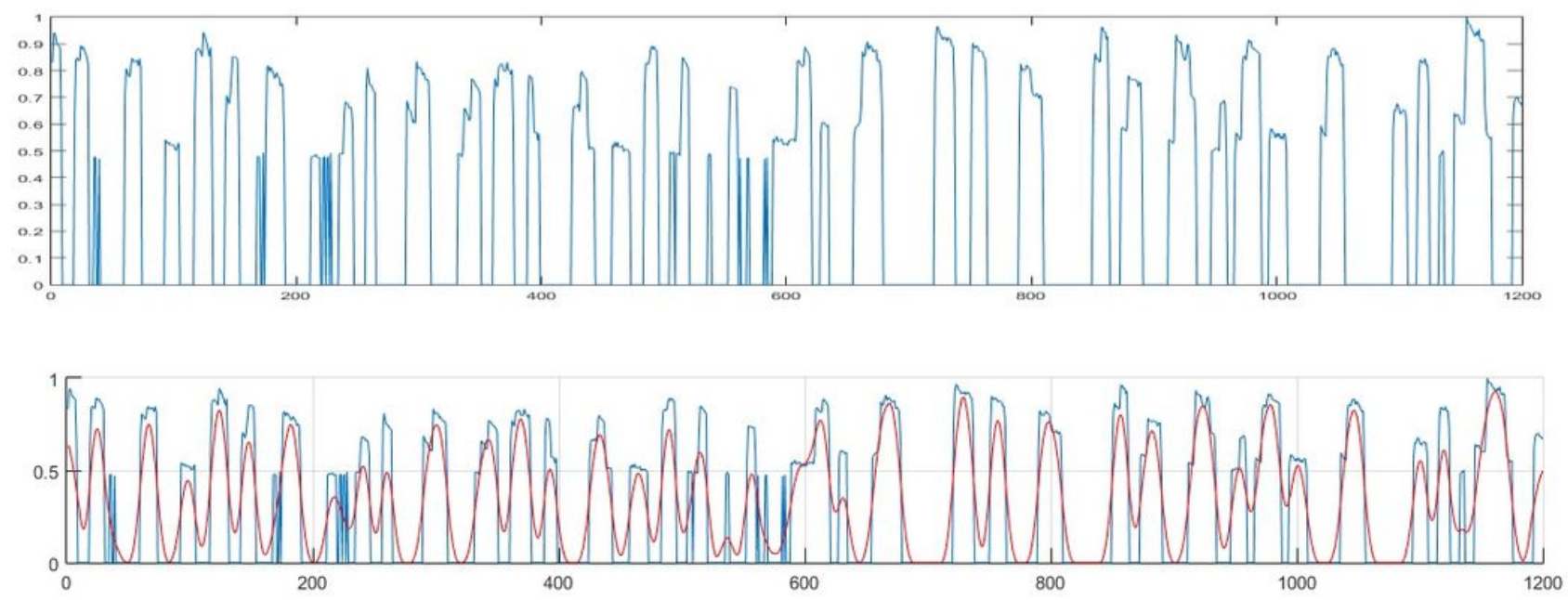

Figure 2

2a. An example of The EOG signals (belong to right eye movement with Visual Distractor) 2b. Application of SSA to the EOG signals 